\title{
La malattia di Fabry in medicina interna: ruolo di febbre e ipertermia nella diagnosi
}

\author{
Elena Verrecchia, Daniela Antuzzi, Francesco De Vito, Manuel Soldato, Donato Rigante, Giovanni Gambaro, Raffaele Manna
}

Centro di Ricerca per le Febbri Periodiche e Malattie Rare, Fondazione Policlinico Gemelli, Università Cattolica del Sacro Cuore, Roma

\begin{abstract}
Fabry disease in internal medicine: the role of fever and hyperthermia in diagnosis
Fabry disease manifests as fever of unknown origin (FUO) in almost $20 \%$ of diagnosed cases. Inflammation parameters are usually variable. Abnormalities in thermal autoregulation can be true when fever is associated with acroparesthesia and paroxysmal abdominal pain as occurs in neuropathies. Otherwise, in the presence of signs and symptoms of chronic inflammation, cytokine secretion and activation is probably due to endothelial and macrophage globotrioside (Gb3) lysosomal storage; proteinuria (less than $1 \mathrm{~g} / 24$ hours) and vascular damage are often present, especially in those patients developing chronic kidney disease and/or cerebrovascular ischemic disease. The diagnosis of Fabry disease can be simplified by investigating the patient's clinical history regarding the presence of angiokeratomas, cornea verticillata, deafness and/or cardiomyopathy together with FUO. FUO onset can delay the diagnosis of Fabry disease and it is crucial to address Fabry disease in the etiopathogenesis of FUO, because early diagnosis of Fabry disease may delay its systemic progression.
\end{abstract}

Keywords: Cytochine, Fabry disease, Fever, Fever of unknown origin (FUO), Thermal autoregolation, Vascular damage

\section{Introduzione}

La febbre è un sintomo importante nei pazienti maschi che presentano il fenotipo classico della malattia di Fabry (MF) sin dall'infanzia (1), in cui spesso è presente all'esordio, assieme alle acroparestesie, e al dolore addominale $(2,3)$. II meccanismo patogenetico della febbre nella MF non è ancora chiaro, ma in un sottogruppo di pazienti è stata associata ad alterazioni dei meccanismi di dispersione del calore del corpo (4), in un altro sottogruppo è stato ipotizzato che l'accumulo lisosomiale di globotriaosylceramide (Gb3) a livello macrofagico e/o endoteliale possa attivare i circuiti citochinici della febbre. In ogni caso, la febbre rappresenta uno dei primi sintomi di $\mathrm{MF}$, che viene più frequentemente frainteso e conduce al ritardo diagnostico di $\operatorname{MF}(2,3)$. A causa della sovrapposizione dello spettro clinico della MF con molte al-

Accepted: March 27, 2017

Published online: September 12, 2017

Indirizzo per la corrispondenza:

Prof. Raffaele Manna

Centro di Ricerca per le Febbri Periodiche e Malattie Rare

Fondazione Policlinico Gemelli

Università Cattolica del Sacro Cuore

Largo F. Vito, 1

00168 Roma

raffaele.manna@unicatt.it tre malattie, la MF è stata descritta come "il nuovo grande impostore" (5), perciò la MF è stata proposta come una rara causa di febbre intermittente di origine sconosciuta (rFUO) (6) da includere nella diagnosi differenziale delle FUO ricorrenti (Tab. I).

Nella nostra ricerca abbiamo studiato la febbre come sintomo di presentazione della MF, tenendo conto sia dei marcatori infiammatori sia valutando l'impatto della febbre sul ritardo diagnostico della malattia.

\section{Metodi}

Abbiamo rivisto la nostra casistica di 58 pazienti (30 maschi, 28 femmine) con diagnosi biochimica e genetica di MF, seguiti per un periodo di 27 anni per cercare la presenza di FUO ricorrenti. Sono stati registrati i tempi di insorgenza e quelli della scomparsa della febbre, i sintomi e i segni associati agli attacchi febbrili, potenzialmente collegabili alla MF; perciò è stata cercata la presenza di acroparestesie, le tipiche crisi addominali, l'intolleranza al caldo, l'ipo-anidrosi, i disturbi gastrointestinali (come dolore addominale, dispepsia e/o diarrea), tinnito, vertigini. Tra i segni sono stati cercati gli angiocheratomi, e la cornea verticillata; negli esami di laboratorio la creatinina, la proteinuria e/o microalbuminuria, e tutti i parametri riferibili a nefropatia. Negli esami strumentali si sono cercate le anomalie dell'elettrocardiogramma (ECG) e/o segni di ipertrofia concentrica rilevabili all'ecocardiografia. Sono stati valutati i marcatori di infiammazione, come la 
TABELLA I - Cause di FUO ricorrenti

\begin{tabular}{|c|c|c|c|}
\hline & Rare & Molto rare & Rarissime \\
\hline Morbo di Addison & Morbo di Still & Malattia di Fabry & Mixoma atriale \\
\hline Cirrosi & $\begin{array}{l}\text { Emboli } \\
\text { di colesterolo }\end{array}$ & $\begin{array}{l}\text { Malattia } \\
\text { di Gaucher }\end{array}$ & TRAPS \\
\hline Febbre da farmaco & Febbre fattizia & $\begin{array}{l}\text { Morbo } \\
\text { di Whipple }\end{array}$ & Istiocitosi X \\
\hline Epatite granulomatosa & Ipertermia abituale & $\begin{array}{l}\text { Sindrome } \\
\text { da iper-IgD }\end{array}$ & $\begin{array}{l}\text { Sindrome } \\
\text { di Schnitzler }\end{array}$ \\
\hline Ipopituitarismo ipotalamico & $\begin{array}{l}\text { Istiocitosi a cellule } \\
\text { di Langerhans }\end{array}$ & $\begin{array}{l}\text { Malattia } \\
\text { di Castleman }\end{array}$ & $\begin{array}{l}\text { Malattia di } \\
\text { Muckle-Wells }\end{array}$ \\
\hline Embolia polmonare & Endocardite subacuta & Colangiti subacute & CINCA/NOMID \\
\hline Tubercolosi & Anemia emolitica & Febbre mediterranea familiare & $\begin{array}{l}\text { Fistola } \\
\text { aorto-enterica }\end{array}$ \\
\hline Ipertrigliceridemia & Linfomi & Mastoiditi croniche & $\begin{array}{l}\text { Malattia di } \\
\text { Erdheim-Chester }\end{array}$ \\
\hline Morbo di Crohn & Sinusite cronica & $\begin{array}{l}\text { Malattia } \\
\text { di Rosai-Dorfman }\end{array}$ & PFAPA \\
\hline Malattia di Behçet & $\begin{array}{l}\text { Ascessi } \\
\text { dentali periapicali }\end{array}$ & Pseudolinfoma & \\
\hline Arterite temporale & Prostatite cronica & $\begin{array}{l}\text { Polmonite da } \\
\text { ipersensibilità (febbre } \\
\text { da umidificatore) }\end{array}$ & \\
\hline \multirow[t]{4}{*}{ Sacroileiti } & Sindrome SAPHO & $\begin{array}{l}\text { Febbre da fumi } \\
\text { di polimeri }\end{array}$ & \\
\hline & & Neutropenia ciclica & \\
\hline & & $\begin{array}{l}\text { Neoplasia } \\
\text { del colon }\end{array}$ & \\
\hline & & $\begin{array}{l}\text { Febbre } \\
\text { da fumi metallici }\end{array}$ & \\
\hline
\end{tabular}

velocità di eritrosedimentazione (VES), la proteina $C$ reattiva e la $\beta_{2}$-microglobulina. Per ogni paziente è stato calcolato il ritardo diagnostico a partire dall'epoca di insorgenza della febbre fino alla diagnosi.

\section{Risultati}

Il nostro studio consente di stabilire che nel 20,7\% (12/58) dei pazienti con MF è presente la febbre e/o ipertermia al momento della comparsa della malattia, siano bambini che adulti. Da notare che il 91,6\% (11/12) dei pazienti con rFUO da noi studiati aveva un fenotipo classico di MF, mentre un solo paziente aveva una malattia a esordio tardivo.

La caratteristica della febbre nei pazienti da noi osservati era quella di una febbre ricorrente, con età media di insorgenza di 7,4 $\pm 2,0$ anni. La specificità degli attacchi febbrili nei 12 pazienti che hanno mostrato presenza di rFUO, andava da una febbre della durata di poche ore dopo esercizio fisico o esposizione al sole (2 pazienti), a un episodio febbrile intermittente al mese della durata di 2-3 giorni occorso durante l'infanzia. Solo il 16,6\% (2 pazienti su 12) riferiva la scomparsa degli attacchi febbrili dopo la crescita, mentre il $50 \%$ dei pazienti (6/12) riferiva la netta scomparsa della febbre dopo la terapia con la sostituzione enzimatica (ERT).

Nella Tabella II sono riportati i sintomi e i segni tipici della MF associati alle rFUO da noi studiate. Gli episodi febbrili sono sempre stati associati a sintomi suggestivi di MF in tutti i pazienti. Tutti lamentavano fatica; 6 pazienti lamentavano dolori addominali, 4 diarrea e 2 avvertivano entrambi. L'83,3\% (10/12) dei pazienti aveva acroparestesie durante gli attacchi febbrili, il 66,6\% (8/12) lamentava intolleranza al calore e il $58,3 \%$ (7/12) aveva anche ipoidrosi, mentre il 41,6\% (5/12) aveva le tipiche crisi di Fabry (crisi dolorose addominali e parestesie dolorose associate alla febbre).

Il coinvolgimento d'organo (proteinuria, ipertrofia miocardica, vertigini e/o tinnito) negli anni successivi al primo episodio febbrile era presente solo in 3 pazienti di cui uno sviluppò una proteinuria con rapida progressione della malattia fino all'insufficienza renale cronica (IRC), mentre 2 pazienti rice- 
TABELLA II - Sintomi e segni di malattia di Fabry nei pazienti con FUO ricorrenti

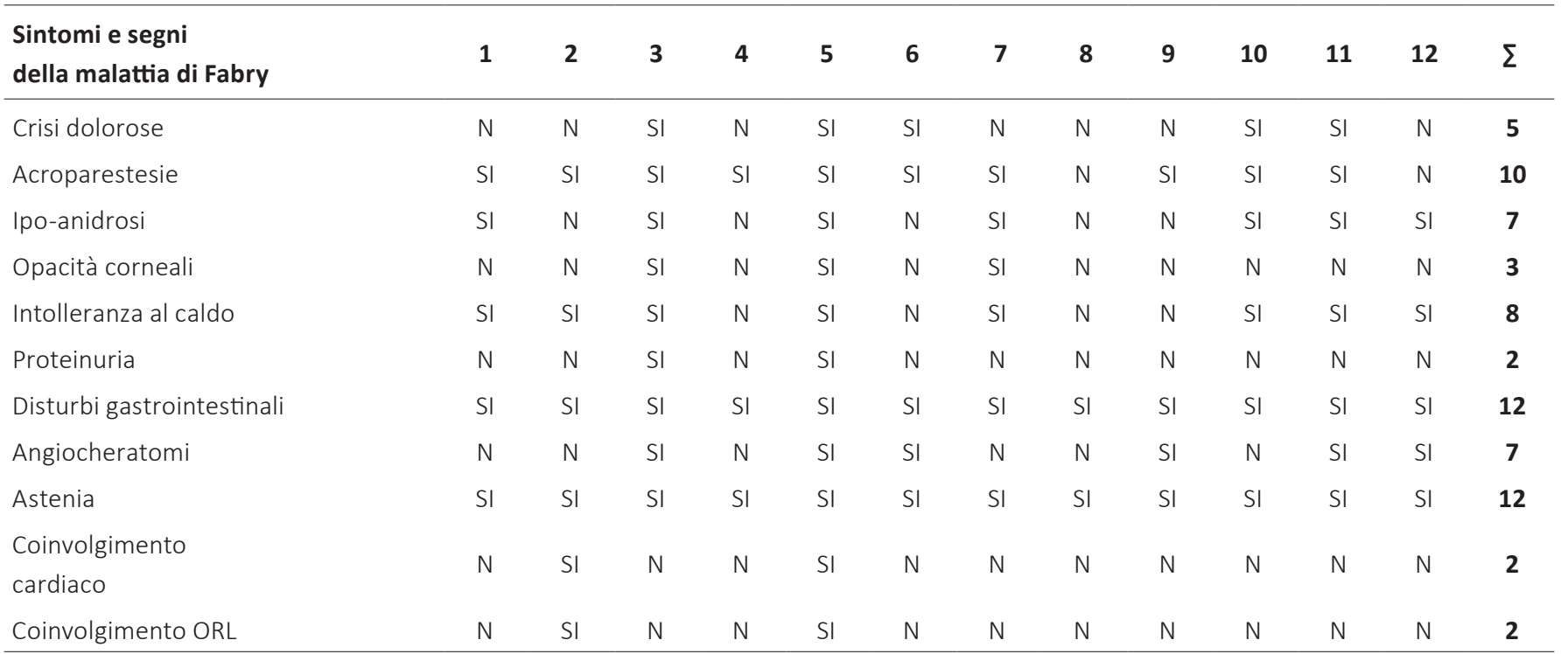

TABELLA III - Caratteristiche della febbre e dei parametri infiammatori

\begin{tabular}{|c|c|c|c|c|c|c|c|c|c|c|c|c|c|}
\hline Febbre & 1 & 2 & 3 & 4 & 5 & 6 & 7 & 8 & 9 & 10 & 11 & 12 & $M$ \\
\hline Età esordio & 8 & 5 & 6 & 6 & 7 & 5 & 6 & 10 & 10 & 10 & 6 & 10 & 7,4 \\
\hline Età scomparsa & 18 & 36 & 10 & 10 & 34 & 20 & 19 & 16 & 30 & 30 & 30 & 18 & 22,6 \\
\hline Recidive/mese & $1-3$ & $<1$ & 1 & 1 & $1-3$ & $<1$ & $<1$ & $<1$ & $<1$ & $<1$ & $<1$ & $<1$ & $1-3$ \\
\hline VES (mm/h) & 10 & 17 & 12 & 3 & 10 & 10 & 6 & 12 & 2 & 6 & 4 & 29 & 12,3 \\
\hline PCR (mg/L) & 2 & 2 & 3 & 2 & 2 & 2 & 3 & 2 & 3 & 14,2 & 2 & 2 & 3,2 \\
\hline$\beta_{2}$-microglobulina (mg/L) & 1,7 & 1,1 & 2,1 & 1,5 & 1,9 & 1,8 & 2,4 & 3,3 & 5 & 1,4 & 1,7 & 2,37 & 2,2 \\
\hline
\end{tabular}

vettero una diagnosi tardiva di MF, nonostante lo sviluppo di manifestazioni evidenti di MF.

Nel complesso infatti il ritardo diagnostico nei nostri 12 pazienti è risultato essere mediamente di $15,8 \pm 12,8$ anni. Il ritardo più lungo si è verificato in un paziente emblematico che ha ricevuto la diagnosi di MF a 48 anni di età, dopo numerose indagini mediche per rFUO iniziate dall'infanzia con RAA (acroparestesie distali interpretate come dolori articolari), nefrite poststreptococcica (proteinuria), e la diagnosi di MF fu ottenuta solo dopo I'identificazione di MF in un altro membro della famiglia.

Nella Tabella III è anche riportata l'associazione tra le caratteristiche della febbre e i principali marcatori infiammatori nei 12 pazienti da noi seguiti. Da notare che il controllo continuativo dei parametri infiammatori non rientrava nel followup di questi pazienti.

\section{Discussione}

Il nostro studio consente di stabilire che nel $20,7 \%$ dei pazienti con MF da noi studiati è presente la febbre e/o iperter- mia al momento della comparsa della malattia, in qualsiasi età siano essi osservati.

I pazienti da noi osservati ricadono in due sottogruppi: nel primo gruppo i marker sistemici di infiammazione risultano assenti durante la febbre e la risposta agli antipiretici è scarsa; questo depone per l'assenza di un meccanismo infiammatorio sottostante, indicando l'origine della febbre in un meccanismo distermico neuropatico. È noto che i depositi di Gb3 riducono la conduzione delle fibre Ac e delta (7) responsabili degli impulsi nocicettivi e termici (8). Questi fenomeni patologici alterano la termoregolazione, che si fonda sia sull'attivazione del metabolismo muscolare che sul meccanismo di vasodilatazione/vasocostrizione cutanea. L'ipo-anidrosi risulta completamente assente solo nel $25 \%$ dei nostri pazienti, ed è stata riportata anche in pazienti con MF senza febbre (9).

La disfunzione endoteliale presente nella $\operatorname{MF}(9,10)$ provoca un'alterazione del tono vascolare fino a fenomeni evidenti di vasocostrizione, spesso scatenati dall'esposizione al freddo; si realizza un fenomeno di Raynaud tipo I, obiettivabile con la capillaroscopia. L'alterazione del tono vascolare 
contribuisce all'alterazione della dissipazione del calore.

In un secondo gruppo di pazienti il pattern di febbre intermittente è correlabile all'aumento degli indici infiammatori presente in alcuni pazienti, coerentemente con la nozione che uno stato infiammatorio cronico è stato dimostrato nella MF per la deposizione sistemica di $\operatorname{Gb} 3(9,11)$ a livello endoteliale e podocitario renale. A questo proposito, Kikumoto et al (12) hanno riportato due casi di MF con manifestazioni neurologiche ed episodi febbrili ripetuti. Si è quindi tentati di ipotizzare che la presenza di marker infiammatori in alcuni pazienti potrebbe essere un indice di maggiore gravità della malattia, nonché un rischio per la progressione della malattia, per eventi ischemici (12), o per comparsa di anemia (13). Due pazienti della nostra serie hanno presentato incrementi dei marcatori di infiammazione, con il coinvolgimento degli organi principali e la progressione della malattia. Pertanto, l'aumento della temperatura corporea osservata nella MF può dipendere in alcuni casi da ipertermia, mentre in altri casi la febbre è correlabile all'infiammazione sistemica, non infettiva e non autoimmune. La disfunzione endoteliale e l'attivazione macrofagica, conseguenti all'accumulo di Gb3, potrebbero essere la fonte di citokine infiammatarie, come I'IL-1 e il TNF-alpha, che giocano un ruolo centrale nel meccanismo febbrile (9).

Il ritardo diagnostico medio nei pazienti febbrili è stato di $15,8 \pm 12$, 8 anni, cioè maggiore di quello sia dei nostri pazienti non febbrili, sia rispetto a quanto riportato in letteratura (13 anni) (14). Poiché i pazienti Fabry comunemente riportano episodi di febbre durante la loro infanzia, dobbiamo ritenere che la febbre rappresenti un sintomo molto precoce, che rimane non diagnosticato $(3,15)$, determinando in tal modo il ritardo diagnostico. Questo dimostra che la diagnosi di MF può essere complessa non solo per la rarità della malattia, ma anche per l'elevato numero di diagnosi differenziali possibili nelle FUO ricorrenti (Tab. I). L'alta prevalenza di acroparestesia 10/12 (=83\%) nei pazienti con febbre rispetto alla frequenza degli angiocheratomi $6 / 12(=50 \%)$ suggerisce che la combinazione di febbre/ ipertermia più acroparestesia è un complesso sindromico facilmente memorizzabile e utilizzabile nella pratica diagnostica.

Un significativo tasso di insorgenza febbrile $(20,7 \%)$, osservato nella nostra coorte, ci porta a concludere che anche la febbre/ipertermia dovrebbe essere inclusa nella diagnosi differenziale delle FUO ricorrenti, al fine di ridurre i tempi di diagnosi e ottimizzare il flusso delle procedure diagnostiche, anche sotto il profilo dei costi.

\section{Ringraziamenti}

I Professori Roberta Ricci, Amelia Morrone e Claudio Feliciani, e la Dottoressa Anna Zampetti hanno collaborato attivamente al progetto "Malattia di Fabry" presso la Fondazione Policlinico Gemelli, Roma.

\section{Disclosures}

Financial support: No financial support was received for this submission.

Conflict of interest: The authors have no conflict of interest.

\section{Bibliografia}

1. Laney DA, Peck DS, Atherton AM, et al. Fabry disease in infancy and early childhood: a systematic literature review. Gen Med. 2015;17(5):323-30.

2. Marchesoni CL, Roa N, Pardal AM, et al. Misdiagnosis in Fabry disease. J Pediatr. 2010;156(5):828-31.

3. Zizzo C, Colomba P, Albeggiani G, et al. Misdiagnosis of familial Mediterranean fever in patients with Anderson-Fabry disease. Clin Genet. 2013;83(6):576-8.

4. Kang WH, Chun SI, Lee S. Generalized anhidrosis associated with Fabry's disease. J Am Acad Dermatol. 1987;17(5 Pt 2):883-7.

5. Lidove O, Kaminsky E, Hachulla E, et al. FIMeD investigators. Fabry disease 'The New Great Imposter': results of the French Observatoire in Internal Medicine Departments (FIMeD). Clin Genet. 2012; 81(6): 571-7.

6. Chao CT, Yang CC, Kao TW. Fabry disease: a rare cause of Fever of unknown origin. Am J Kidney Dis. 2012;59(1):161-2.

7. Torvin M $\varnothing$ ller A, Staehelin Jensen T. Neurology in Fabry disease. Clin Ther. 2008; 30(Suppl B): S47-9.

8. Samuelsson K, Kostulas K, Vrethem M, Rolfs A, Press R. Idiopathic small fiber neuropathy: phenotype, etiologies, and the search for Fabry disease. J Clin Neurol. 2014;10(2):108-18.

9. De Francesco PN, Mucci JM, Ceci R, Fossati CA, Rozenfeld PA. Fabry disease peripheral blood immune cells release inflammatory cytokines: role of globotriaosylceramide. Mol Genet Metab. 2013;109(1):93-9.

10. Satoh K. Globotriaosylceramide induces endothelial dysfunction in Fabry disease. Arterioscler Thromb Vasc Biol. 2014;34(1):2-4.

11. Dubost JJ, Sauvezie B, Galtier B, Tixeron J, Rampon S. Fabry's disease. Rare etiology of a long-term inflammatory syndrome. Apropos of a case. Rev Rhum Mal Osteoartic. 1986;53(10):525-8.

12. Kikumoto $\mathrm{Y}$, Kai $\mathrm{Y}$, Morinaga $\mathrm{H}$, et al. Fabry disease exhibiting recurrent stroke and persistent inflammation. Intern Med. 2010;49(20):2247-52.

13. Kleinert J, Dehout F, Schwarting $A$, et al. Anemia is a new complication in Fabry disease: data from the Fabry Outcome Survey. Kidney Int. 2005;67(5):1955-60.

14. Thomas AS, Mehta AB. Difficulties and barriers in diagnosing Fabry disease: what can be learnt from the literature? Expert Opin Med Diagn. 2013;7(6):589-99.

15. Abreo K, Oberley TD, Gilbert EF, Opitz JM, Updike SJ. Clinicopathological conference: a 29-yr-old man with recurrent episodes of fever, abdominal pain, and vomiting. Am J Med Genet. 1984;18(2):249-64. 